\title{
Retroperitoneal lymphadenopathy: an extrahepatic feature of chronic active hepatitis
}

\author{
Tony Hayek, Arie Markel, Moshe Goldfeld ${ }^{1}$, Yehudit Ben-Arie ${ }^{2}$ and \\ Gerald J. Brook
}

Departments of Internal Medicine D, ${ }^{1}$ Radiology and ${ }^{2}$ Pathology, Rambam Medical Center, Haifa, 31096 Israel

\begin{abstract}
Summary: We report a patient with chronic active hepatitis in whom one of the initial findings was retroperitoneal lymphadenopathy, detected by abdominal ultrasound examination. Extrahepatic presenting findings of chronic active hepatitis may include arthritis, urticaria, pleurisy and pericarditis, while abdominal lymphadenopathy has been only rarely described. Chronic active hepatitis should be included in the differential diagnosis of abdominal lymphadenopathy.
\end{abstract}

\section{Introduction}

Chronic active hepatitis (CAH) is a disorder characterized by continuing hepatic necrosis, active inflammation and fibrosis, which may lead to, or be accompanied by, liver failure, cirrhosis and death. ${ }^{1}$ Many agents may initiate chronic active hepatitis, including the virus of hepatitis $B$, hepatitis $C$ and hepatitis $D$, and the non-A non-B hepatitis virus. Many CAH patients have an autoimmune hepatitis which often is associated with other immune disorders such as thyroiditis, ulcerative colitis, Coombs' positive haemolytic anaemia, and Sjogren's syndrome.

The clinical spectrum of CAH extends from asymptomatic illness to fatal hepatic failure. Recurrent jaundice, malaise, anorexia and lowgrade fever are common throughout the course of the illness, but in some patients extrahepatic features dominate and liver disease is entirely unsuspected. Extrahepatic presenting features may include arthralgia, arthritis, urticaria, rash, pleurisy and pericarditis. ${ }^{2,3}$ Abdominal lymphadenopathy accompanying CAH has been described rarely. ${ }^{4,5}$ We present a patient with $\mathrm{CAH}$ in whom retroperitoneal lymphadenopathy was discovered on the initial imaging evaluation.

\section{Case report}

A 41 year old man was admitted for evaluation of malaise, fatigue and myalgia. The liver was mildly

Correspondence: A. Markel, M.D.

Accepted: 16 September 1993 enlarged. Results of laboratory tests were: haemoglobin $15.4 \mathrm{~g} / \mathrm{dl}$, white blood cell count of $10 \times 10^{9} /$ 1 with a normal differential count. Electrolytes, blood urea nitrogen, amylase, calcium, phosphate, prothrombin time and platelet count were normal. The alkaline phosphatase was $125 \mathrm{U} / 1$ (normal range 30-110), alanine aminotransferase (ALT) $134 \mathrm{U} / 1$ (NR 5-50), aspartate aminotransferase (AST) $101 \mathrm{U} / 1$ (NR 5-45); total protein, albumin, total bilirubin and lactate dehydrogenase were normal. Anti-mitochrondrial and anti-smooth muscle antibodies were negative. Tests for hepatitis A, hepatitis B, Epstein-Barr virus, and cytomegalovirus were negative.

Abdominal ultrasound scan demonstrated normal liver parenchyma and retroperitoneal lymphadenopathy. A computed tomographic (CT) scan of the abdomen showed mild hepatomegaly, normal liver parenchyma and enlarged lymph nodes involving the retroperitoneal, paraaortic and interaortocaval chains (Figure 1). A gallium scan and chest X-ray were normal. An upper gastrointestinal barium study was normal. A bone marrow biopsy was normal. A liver needle biopsy showed lymphoplasmacytic infiltration of the portal spaces with areas of piecemeal necrosis, erosion of the limiting plate, and early fibrosis of the portal tract, diagnostic of chronic active hepatitis (Figure 2).

The patient was started on prednisone, $30 \mathrm{mg} /$ day with improvement of his symptoms and of the liver function tests, and reduction in the size of the abdominal lymph nodes as demonstrated by $\mathrm{CT}$ scan (Figure 3). He continues to do well on prednisone, $10 \mathrm{mg} /$ day. 


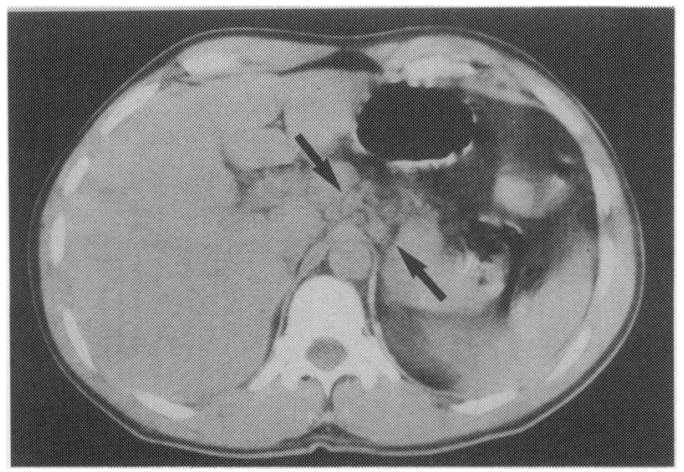
moderately enlarged coeliac trunk lymph nodes (arrows).

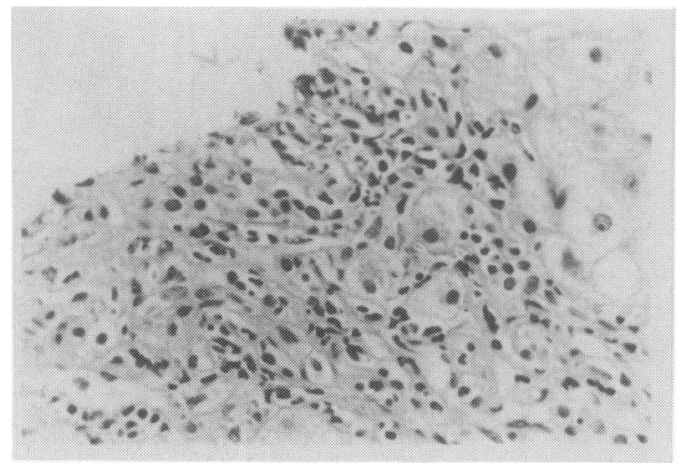

Figure 2 Liver biopsy showing intense mononuclear inflammatory infiltrate in the portal area with foci of piecemeal necrosis on the left-hand side $(H \& E, \times 500)$.

\section{Discussion}

The presence of abdominal lymphadenopathy in association with chronic active hepatitis is rare. Most commonly, abdominal lymphadenopathy is caused by lymphoma and metastatic disease. Benign causes have been reported in up to $6 \%$ of patients. ${ }^{6}$ Forsberg et al. ${ }^{4}$ have reported adenopathy in the hepatoduodenal ligament detected by ultrasonography in patients with diffuse, chronic immune liver diseases (such as primary biliary cirrhosis, sclerosing cholangitis and hepatitis B surface antigen positive, and autoimmune CAH). Gore et al. have also reported retroperitoneal adenopathy detected by abdominal CT scan in patients with CAH. ${ }^{5}$ In our patient other causes of lymphadenopathy such as infections or malignancy were ruled out by serological tests, liver and bone marrow biopsies, and by ultrasound and
Figure 1 Prior to treatment. CT scan shows a cluster of

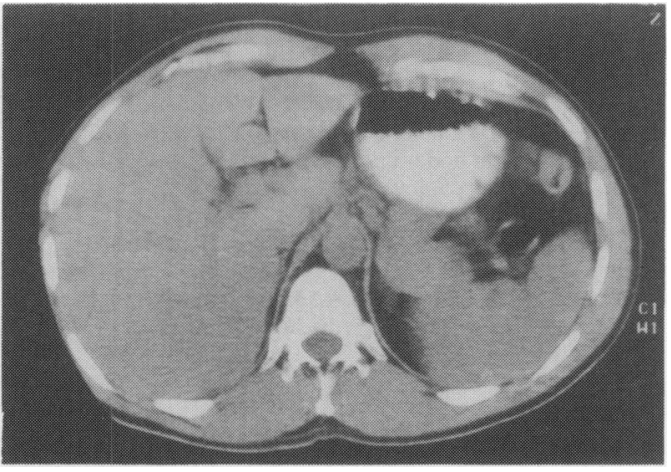

Figure 3 After treatment. CT scan at the same plane shows partial regression of the lymphadenopathy.

CT scanning of the abdomen, which demonstrated no pathological process except lymphadenopathy.

The lymphadenopathy in CAH is presumably due to reactive hyperplasia combined with direct spread of the hepatic inflammatory process to the retroperitoneal lymph nodes which drain the liver., ${ }^{4,5}$ Our patient had significant clinical and laboratory improvement on steroid therapy.

Patients with pathological liver function tests are often examined with ultrasound or CT scan as a first step in establishing a diagnosis. Fatty liver or cirrhosis can usually be detected ${ }^{7,8}$ while the diagnosis of CAH is often difficult or impossible based on examination of the liver alone. The detection of abdominal lymphadenopathy in the same patient could make the diagnosis more feasible.

The features of the lymph nodes on ultrasound or CT scan such as size, number and distribution are important in the evaluation of abdominal lymphadenopathy. Subramanyam et al. ${ }^{9}$ reported abdominal lymphadenopathy in intravenous drug addicts as a result of reactive hyperplasia, various infections, and neoplasms. In these patients small nodes $(<1.5 \mathrm{~cm})$ located in the porta hepatis, coeliac axis and peripancreatic regions are usually due to reactive hyperplasia, while large nodes $(>1.5 \mathrm{~cm})$ located in the mid or lower retroperitoneum below the coeliac axis or within the mesentery should be considered as due to a pathological process other than benign hyperplasia. ${ }^{9}$

In conclusion CAH should be included in the differential diagnosis of abdominal lymphadenopathy, particularly if liver function tests are abnormal. In addition, abdominal lymph nodes may serve as a marker for disease activity in the follow-up of these patients.

\section{References}

1. Czaja, A.J. Natural history, clinical features and treatment of autoimmune hepatitis. Semin Liver Dis 1984, 4: 1-12.
2. Pear, B.L. Radiologic recognition of extrahepatic manifestations of hepatitis B antigenemia. AJR 1981, 137: 135-140. 
3. Strober, W. \& James, S.P. The immunopathogenesis of gastrointestinal and hepatobiliary diseases. JAMA 1987, 258: 2962-2969.

4. Forsberg, L., Floren, C.H., Hederstrom, E. et al. Ultrasound examination in diffuse liver disease. Clinical significance of enlarged lymph nodes in the hepato-duodenal ligament. Acta Radiol (Diagn) (Stockh) 1987, 28: 281-284.

5. Gore, R.M., Vogelzang, R.L. \& Nemcek, A.A. Lymphadenopathy in chronic active hepatitis: CT observations. $A J R$ 1988, 151: 75-78.

6. Deutsch, S.J., Sandler, M.A. \& Alpern, M.B. Abdominal lymphadenopathy in benign diseases: CT detection. Radiology 1987, 163: 335-338.
7. Foster, K.J., Dewbury, K.C., Griffith, A.H. et al. The accuracy of ultrasound in the detection of fatty infiltration of the liver. Br J Radiol 1980, 53: 440-442.

8. Taylor, K.J.W., Carpenter, D.A., Hill, C.R. et al. Gray scale ultrasound imaging. The anatomy and pathology of the liver. Radiology 1976, 119: 415-423.

9. Subramanyam, B.R., Balthazar, E.J., Horil, S.C. et al. Abdominal lymphadenopathy in intravenous drug addicts: sonographic features and clinical significance. $A J R$ 1985, 144: 917-920. 